\title{
Moderate Secondary Tricuspid Regurgitation: Does It Worth More Time to Conserve?
}

\author{
Montaser Elsawy Abd Elazizi,2, Ahmed Labib Dokhan1, \\ Awny Gamal Shalaby33, Hesham Hassan Ahmed1, \\ Mohammed Ahmed El-Hag-Aly ${ }^{1}$, Mohammed Fawzy Eltaweel ${ }^{1}$ \\ ${ }^{1}$ Cardithoracic Surgery Department, Faculty of Medicine, Menoufia University, Menoufia, Egypt \\ ${ }^{2}$ Faculty of Medicine, Jazan University, Jazan, KSA \\ ${ }^{3}$ Cardiology Department, Faculty of Medicine, Menoufia University, Menoufia, Egypt \\ Email: montaserabdelaziz5@gmail.com
}

How to cite this paper: Elaziz, M.E.A., Dokhan, A.L., Shalaby, A.G., Ahmed, H.H., El-Hag-Aly, M.A. and Eltaweel, M.F. (2021) Moderate Secondary Tricuspid Regurgitation: Does It Worth More Time to Conserve? Open Journal of Thoracic Surgery, 11, 59-68.

https://doi.org/10.4236/ojts.2021.113008

Received: July 4, 2021

Accepted: August 10, 2021

Published: August 13, 2021

Copyright $\odot 2021$ by author(s) and Scientific Research Publishing Inc. This work is licensed under the Creative Commons Attribution International License (CC BY 4.0).

http://creativecommons.org/licenses/by/4.0/

\section{(c) (i) Open Access}

\begin{abstract}
Background: Concomitant repair of moderate secondary tricuspid regurgitation (STR) during mitral valve surgery is still subject of controverse. This research aims to study the early and mid-term postoperative influence of tricuspid repair on the clinical outcomes and right ventricular functions. Methods: This is a retrospective cohort study of 92 patients with moderate STR who underwent tricuspid valve repair between October 2014 and December 2017, in the cardiothoracic surgery department, Menoufia University Hospital. Patients were assessed clinically and by echocardiography at one month and after 24 months postoperatively for the degree of tricuspid regurgitation (TR), right ventricular diameter and tricuspid annular plane systolic excursion (TAPSE). Results: In this study, mean age of patients was $58.33 \pm 4.06$ years. We had a significant improvement in the grade of TR from preoperative period to follow up data observed at 1-month postoperative and 2-years follow-up $(\mathrm{P}=$ 0.0001). After one month postoperatively, no TR was detected in 74 cases (80.4\%), and in $71(84.5 \%)$ after two years. Mean right ventricular diameter decreased significantly from $23 \pm 3.32 \mathrm{~mm}$ preoperatively to $21.9 \pm 3 \mathrm{~mm}$ at 2-years postoperatively. An increase of TAPSE was from $19.96 \pm 3.7 \mathrm{~mm}$ preoperatively to $20.3 \pm 3.9 \mathrm{~mm}$ at 1 -month postoperative $(\mathrm{p}=0.55)$ and there was significant increase in late postoperative value. Conclusion: We highly recommend repair for moderate TR during mitral valve surgery to avoid progression of TR. Tricuspid repair was able to reduce the grade of regurgitation after two years of surgery and improved right ventricular functions.
\end{abstract}




\section{Keywords}

Functional Tricuspid Regurgitation, Tricuspid Valve Repair

\section{Introduction}

Functional tricuspid regurgitation (FTR) is a disease that has only recently been recognized as worthy of surgeons' attention. Nonetheless, no clear agreement has been reached on when and how FTR should be corrected. Furthermore, the early return of FTR has made surgeons hesitant to expand FTR repair. Patients with moderate or severe FTR had a significantly lower survival rate than patients without or with only mild FTR [1]. FTR is even less than mild, and if left untreated, it will be escalated by two grades after nearly five years [2].

The majority of tricuspid regurgitation patients have secondary tricuspid regurgitation, which is not caused by primary organic tricuspid valve disease. The $\mathrm{RV}$ and annular dilatation or tethering are associated with secondary TR pathophysiology (even with little annular dilatation). This is most commonly caused by left-side valvular disease, but it can also arise as a result of primary RV pathology or pulmonary hypertension. TR is caused by a loss of contraction of the myocardium surrounding the annulus, hence atrial fibrillation can play a role. There is a failure of coaptation and tethering of the leaflets with increasing enlargement of the ventricle and annulus [3].

Secondary tricuspid regurgitation (STR) is caused by a valvular lesion on the left side of the heart, most often mitral valve disease (MVD). As high pulmonary pressure occurs, it can lead to dilation of the right ventricle and annulus, as well as tricuspid valve (TV) tenting. The tricuspid leaflets have a typical morphology but do not coapt well. However, TR does not occur in all patients with pulmonary hypertension; the cause appears to be multifactorial and linked to other factors such as atrial fibrillation (AF) [4] [5]. We decided to treat STR aggressively in patients undergoing mitral valve (MV) surgery. In this study, we report our experience in evaluating the midterm results of our surgical strategy and standardizing surgical treatment principles. Our research focused on tricuspid repair in patients with moderate TR.

\section{Materials and Methods}

\subsection{Study Design}

This retrospective study was carried out on 92 patients who were candidates for mitral valve surgery and had moderate STR. They underwent tricuspid valve repair with ring annuloplasty technique between October 2014 and December 2017, in the cardiothoracic surgery department, Menoufia University Hospital. Our local institutional review board approved this research under number (2014/9/113), and waived the requirement for patient's consent. Patients with TR who had particular organic leaflet or subvalvular deformities, which includes prolapse, Ebstein anomaly, rheumatic, infective endocarditis, and who had con- 
current surgical interventions other than mitral and tricuspid valve replacement, have been excluded from the study.

\subsection{Operative Technique}

Through median sternotomy, and after establishment of cardiopulmonary bypass, we approached the mitral valve which was replaced in 80 patients and the remaining was repaired. Moderate hypothermia of $32^{\circ} \mathrm{C}$ was achieved, and tepid blood antegrade cardioplegia was used after ascending aortic cross-clamping.

A 3D annuloplasty ring (Medtronic, California, US) was used in all cases. The first suture was placed starting from the anteroseptal commissure and the last one was placed at the posteroseptal commissure. The ring size was determined by the length of the septal leaflet.

We followed up our patients through two years period by echocardiography, and we analyzed preoperative, 1-month, and 2-years postoperative echocardiograms. The same operator who did and interpreted the echocardiography was (A. Sh). GE VIVID S5 Norton Norway echocardiography transducer 1.7 - 4 $\mathrm{mHZ}$ was used. The purpose of our echocardiographic study was to grade tricuspid regurgitation as mild, moderate, or severe when the distal jet area was less than $5 \mathrm{~cm}^{2}, 5-10 \mathrm{~cm}^{2}$, or greater than $10 \mathrm{~cm}^{2}$ respectively.

We used the tricuspid annular plane systolic excursion (TAPSE) to help in estimation of the right ventricular function. It is considered normal if it measures $1.7 \mathrm{~cm}$. The diameter of the right ventricle could be measured at the level of mid cavity [6].

TR grade at one month, two years after surgery and hospital mortality were the primary endpoints. Hospital readmission due to right-sided heart failure and two-year survival was the secondary endpoints of this research.

\subsection{Statistical Analysis}

Statistical significance was determined with an IBM compatible computer and IBM SPSS statistics version 19. Quantitative variables were expressed as mean and standard deviation (Mean, SD), whereas qualitative data were expressed as a number ( $\mathrm{n}$ ) or a percentage (\%) based on relevance. The paired student $\mathrm{t}$-test was used to determine the significance of quantitative variables. We considered probability values less than 0.05 as statistically significant.

\section{Results}

\section{Demographic and Clinical Data}

Regarding demographic data (Table 1), male distribution was 34 cases (37\%), while female distribution was 58 cases (63\%). Mean age was $58.33 \pm 4.06$ years. 54 cases $(58.7 \%)$ had sinus rhythm, while 38 cases $(41.3 \%)$ had arrhythmia.

Pre-operative echocardiography data of the studied patients in Table 2, showed all patients had moderate TR. The mean EF was 59\%. Mean tenting area was $1.30 \pm 0.66 \mathrm{~cm}^{2}$, mean PAP was $45.9 \pm 6.27 \mathrm{mmHg}$, Mean right ventricle (RV) size was $23 \pm 3.32 \mathrm{~mm}$. TAPSE was $19.96 \pm 3.7 \mathrm{~mm}$, and right atrium (RA) 
Table 1. Demographic characteristics and anthropometric measurements of studied patients.

\begin{tabular}{cc}
\hline Variables & (No. $=\mathbf{9 2})$ \\
\hline Age (years) Mean $\pm \mathrm{SD}$ & $58.33 \pm 4.06$ \\
Gender & No. $(\%)$ \\
Male & $34(37)$ \\
Female & $58(63)$ \\
BMI $\left(\mathrm{Kg} / \mathrm{m}^{2}\right)$ Mean $\pm \mathrm{SD}$ & $24.7 \pm 2.26$ \\
BSA $\left(\mathrm{m}^{2}\right)$ Mean $\pm \mathrm{SD}$ & $1.55 \pm 0.21$ \\
Preoperative arrhythmia & No. $(\%)$ \\
Sinus rhythm & $54(58.7)$ \\
Arrhythmia & $38(41.3)$ \\
\hline
\end{tabular}

(BMI: body mass index, BSA: body surface area).

Table 2. Pre-operative echocardiography data of the studied patients.

\begin{tabular}{cc}
\hline Variables & (No. = 92) \\
\hline Degree of TR & No. (\%) \\
-Moderate & $92(100 \%)$ \\
EF (\%) Mean \pm SD & $59 \pm 5.9$ \\
Tenting Area $\left(\mathrm{cm}^{2}\right)$ Mean \pm SD & $1.30 \pm 0.66$ \\
TVCD $(\mathbf{m m})$ Mean \pm SD & $2.8 \pm 0.65$ \\
PAP $(\mathbf{m m H g})$ Mean \pm SD & $45.9 \pm 6.27$ \\
RV size $(\mathbf{m m})$ Mean \pm SD & $23 \pm 3.32$ \\
TAPSE $(\mathbf{m m})$ Mean \pm SD & $19.96 \pm 3.7$ \\
RA $(\mathbf{c m})$ & No. $\%$ \\
Normal & $27(29.35)$ \\
Dilated & $65(70.65)$ \\
\hline
\end{tabular}

EF: ejection fraction, TVCD: Tricuspid valve coaptation depth, PAP: pulmonary artery pressure, RV: right ventricle, TAPSE: Tricuspid annular plane systolic excursion).

was dilated in 65 (70.65\%) patients.

\section{Echocardiography Follow-up}

Regarding postoperative follow up echocardiography (Table 3 and Table 4), We found the mean preoperative ejection fraction was $59.77 \pm 5.9$ and increasd to $60.4 \pm 3.51$-month postoperative and partially increased to $60.2 \pm 4.5$ at 2 -years postoperative follow up ( $\mathrm{P}=0.38$ and 0.58 respectively).

The mean tenting area showed significant reduction from $1.30 \pm 0.66 \mathrm{~cm}^{2}$ preoperative to $1.1 \pm 0.7 \mathrm{~cm}^{2}$ at 1-month postoperative follow up, and $1.12 \pm 0.56 \mathrm{~cm}^{2}$ 2 -years postoperative values ( $\mathrm{P}=0.048,0.048$ respectively). There was a significant regression in pulmonary artery pressure $(\mathrm{PAP})$ between preoperative, postoperative 1-month echocardiograms and those obtained at 2-years follow up $(\mathrm{P}=0.0001)$.

We had a significant improvement in the grade of TR from preoperative period to follow up data obtained at 1-month postoperative and 2-years follow up 
$(\mathrm{P}=0.0001)$. After one month postoperatively, no TR was detected in 74 cases (80.4\%), and in 71 (84.5\%) after two years. (Table 3 and Table 4, Figure 1).

Table 3. Comparison between preoperative and 1-month postoperative echocardiography parameters.

\begin{tabular}{cccc}
\hline \multirow{2}{*}{ Variables } & Preoperative (No. = 92) & 1-month Postoperative (No. = 92) & \multirow{2}{*}{ P value } \\
\cline { 2 - 3 } & Mean \pm SD & Mean \pm SD & 0.38 \\
EF (\%) & $59.77 \pm 5.9$ & $60.4 \pm 3.5$ & \\
TR & No. \% & No. \% & $0.0001^{* *}$ \\
No & 0 & $74(80.4)$ & \\
Mild & 0 & $10(10.9)$ & 0.048 \\
Moderate & $92(100)$ & $8(8.7)$ & 0.02 \\
Tenting Area (cm $)$ & $1.30 \pm 0.66$ & $1.1 \pm 0.7$ & $0.0001^{* *}$ \\
TVCD (mm) & $2.8 \pm 0.65$ & $2.6 \pm 0.5$ & 0.25 \\
PAP ( mmHg) & $45.9 \pm 6.27$ & $35.5 \pm 4.5$ & 0.55 \\
RV size ( mm) & $23 \pm 3.32$ & $22.5 \pm 2.5$ & \\
TAPSE (mm) & $19.96 \pm 3.7$ & $20.3 \pm 3.9$ & $0.0001^{* *}$ \\
RA (cm) & No. \% & No. \% & \\
Normal & $27(29.35)$ & $59(64.13)$ & $33(35.86)$ \\
Dilated & $65(70.65)$ & & \\
\hline
\end{tabular}

EF: ejection fraction, PAP: pulmonary artery pressure, RV: right ventricle, TAPSE: Tricuspid annular plane systolic excursion, RA: right atrium). ${ }^{*}$ highly significant difference.

Table 4. Comparison between preoperative and 2-years postoperative echocardiography parameters.

\begin{tabular}{cccc}
\hline \multirow{2}{*}{ Variables } & Preoperative (No. = 92) & 2-years Postoperative (No. = 84) & \multirow{2}{*}{ P value } \\
\cline { 2 - 3 } & Mean \pm SD & Mean \pm SD & 0.58 \\
EF (\%) & $59.77 \pm 5.9$ & $60.2 \pm 4.5$ & \\
TR & No. \% & No. \% & $0.0001^{* *}$ \\
No & $0(0)$ & $71(84.5)$ & \\
Mild & $0(0)$ & $8(9.5)$ & 0.048 \\
Moderate & $92(100)$ & $5(6)$ & 0.012 \\
Tenting Area (cm $)$ & $1.30 \pm 0.66$ & $1.12 \pm 0.56$ & $0.0001^{* *}$ \\
TVCD (mm) & $2.8 \pm 0.65$ & $2.55 \pm 0.7$ & 0.019 \\
PAP ( mmHg) & $45.9 \pm 6.27$ & $33 \pm 5$ & 0.001 \\
RV size ( mm) & $23 \pm 3.32$ & $21.9 \pm 3$ & \\
TAPSE (mm) & $19.96 \pm 3.7$ & $21.5 \pm 2.45$ & \\
RA (cm) & No. \% & No. \% & \\
Normal & $27(29.35)$ & $60(71.4)$ & $0.0001^{* *}$ \\
Dilated & $65(70.65)$ & $24(28.6)$ & \\
\hline
\end{tabular}

EF: ejection fraction, PAP: pulmonary artery pressure, RV: right ventricle, TAPSE: Tricuspid annular plane systolic excursion, RA: right atrium). 


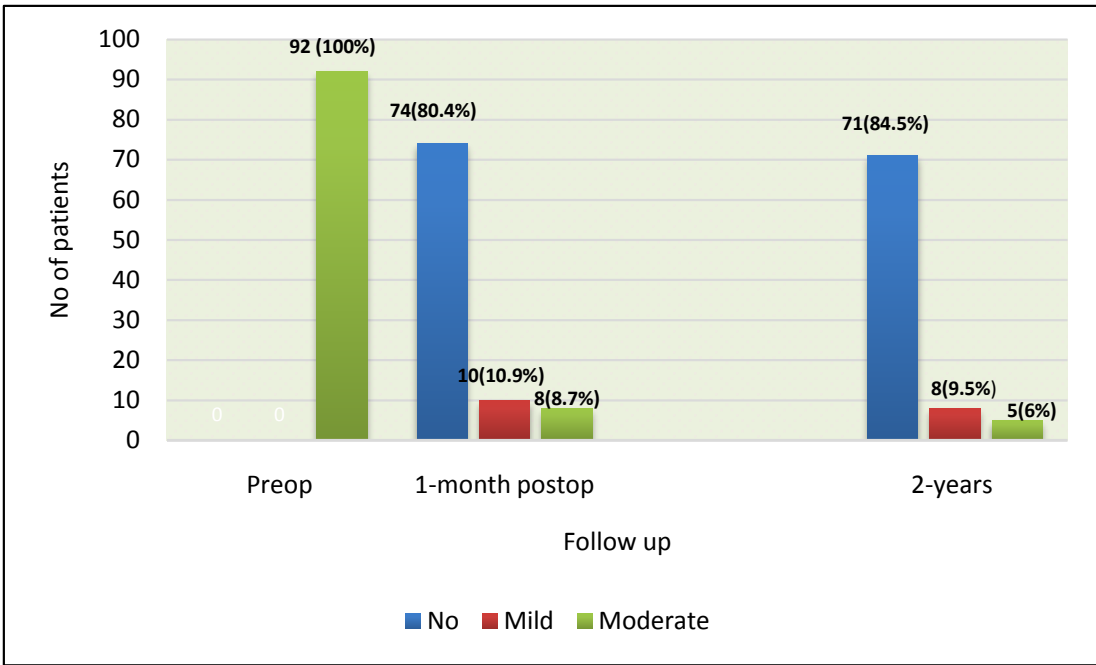

Figure 1. Degree of TR in preoperative, 1-month postoperative and 2-years postoperative follow-up.

Mean right ventricular diameter decreased from preoperative values $23 \pm 3.32$ $\mathrm{mm}$ to postoperative follow up at 1-month $22.5 \pm 2.5 \mathrm{~mm}$ and decreased significantly at 2-years to $21.9 \pm 3 \mathrm{~mm},(\mathrm{P}=0.019)$.

An increase of TAPSE value from $19.96 \pm 3.7 \mathrm{~mm}$ preoperatively to $20.3 \pm 3.9$ $\mathrm{mm}$ at 1 -month postoperative $(\mathrm{P}=0.55)$ and there was significant increase in late postoperative value $(\mathrm{P}=0.001)$.

We lost the follow up of eight patients after two years, so we had 84 (91.3\%) patients with their echo-data were complete. One patient died due to cerebral haemorrhage after four months of discharge. The second patient died after six months of discharge due to cardiac arrest which happened because he developed cardiac tamponade as his INR was 7. Resuscitative measures failed to save his life. The rest of lost patients for follow up did not come to the outpatient clinic at a proper time and were not adherent to follow up regularly, so we excluded them from analysis at 2-years echocardiography follow up.

\section{Discussion}

We reported that extensive treatment of STR of any grade resulted in an early reduction of the STR grade that was sustained after 24 months. Surgical management of moderate STR has been a subject of controversy. Moreover in current era of minimal invasive techniques may focus attention on a single valve, ignoring mild to moderate TR, even though the TV is accessible.

Yilmaz and colleagues [7] investigated changes in the degree of STR in 699 patients who underwent MV repair for degenerative MV disease. Their findings showed that TR progression is unusual in patients undergoing MV repair, and that coexisting TR has no effect on late mortality.

In our study we recommended measurement of tricuspid valve dimensions intraoperatively even for mild to moderate TR to assess if the valve could be repaired or not, and which technique could be suitable. This idea has been widely 
studied by Dreyfus and colleagues [2] who suggested that the TV annulus be measured during left heart valve surgery and that tricuspid dilatation be corrected regardless of TR severity because TV disease is correlated with tricuspid dilatation rather than TR degree. The tricuspid annular diameter was measured intraoperatively from the anteroseptal commissure to the anteroposterior commissure. In their study, patients with a tricuspid annular dimension $70 \mathrm{~mm}$ underwent tricuspid annuloplasty, which improved functional status.

We had a significant improvement in the grade of TR from preoperative period to follow up data observed at 1-month postoperative and 2-years follow up $(\mathrm{P}=0.0001)$. After one month postoperatively, no TR was detected in 74 cases (80.4\%), and in $71(84.5 \%)$ after two years.

Nath and colleagues [1] examined echocardiography of 5223 patients at one laboratory over a four-year period. TR occurred in 88.5 percent of cases, with 15.5 percent having moderate or greater TR. After adjusting for left ventricular ejection fraction, inferior vena cava size, and right ventricular size and function, these patients had a 4-year survival worse than that of patients without TR.

Our results came in agreement with Calafiore et al., [8] who conducted a report on 50 patients who had mild to severe FTR. The TR grade in the treated group had decreased from $3.1 \pm 0.7$ to $0.7 \pm 0.7(\mathrm{P}=0.001)$ at one year postoperatively, while it had remained stable in the untreated group. (from $2.2 \pm 0.4$ to $2.3 \pm 0.9, \mathrm{P}$ was not significant). Thus, tricuspid repair provided good midterm results.

Ring annuloplasty was effective in the early postoperative period; however, after 6-months of follow-up, it was associated with less residual regurgitation and better right ventricular remodeling in patients with severe functional tricuspid regurgitation than the De Vega procedure [9].

Saran and Dearani [10] decided that there are several aspects of tricuspid repair can result in complications, such as coronary or conduction system injury. When the primary indication for surgery is TR, it is crucial to achieve an excellent result (less than mild TR). But on contorary to secondary TR (as in case of mitral regurgitation) where the degree of TR is expected to decrease after correction of the left-sided pathology and accepting a less than perfect result (moderate TR) would be the goal.

Ren et al., [11] reported FTR recurrence including Mild degree was 23.5\%. Meanwhile, moderate to severe FTR recurrence was $2.5 \%$.

Our study showed increase of TAPSE value from $19.96 \pm 3.7 \mathrm{~mm}$ preoperatively to $20.3 \pm 3.9 \mathrm{~mm}$ at 1 -month postoperative $(\mathrm{P}=0.55)$ and there was significant increase in late postoperative value $(P=0.001)$, so it affected the RV size which decreased in the postoperative follow up periods especially after two years $[\mathrm{P}=0.019]$. This was in agreement with Hamdy and colleagues [9] where they measured right ventricular function by TAPSE and reported significant improvement in the early postoperative period and after six months in both groups $(\mathrm{P}<0.005)$. PAP improved in the early postoperative period and after six months in both groups. 
TAPSE-measured right ventricular function improved in the early postoperative period and after two years. The reduction in PAP and RV afterload postoperatively may explain the immediate improvement in TAPSE in the early postoperative period.

A study by Desai and colleagues [12] who followed up right ventricular function after mitral valve surgery with or without tricuspid valve repair, and found that in patients with severe TR and mitral valve disease, mitral valve repair alone was accompanied by improvement in TR and right ventricular function early after surgery, the same as mitral valve surgery with concomitant tricuspid valve repair is.

Accurate quantification of TR severity, identification of TR mechanisms, and quantification of RV dysfunction are critical points for preprocedural imaging of TV intervention [13]. One of these is tenting area measurement preoperatively and follow up postoperatively. Our study demonstrated significant decrease in the tenting area from $1.30 \pm 0.66 \mathrm{~cm}^{2}$ preoperatively to $1.1 \pm 0.7 \mathrm{~cm}^{2}$ at 1-month follow up ( $\mathrm{p}=0.048$ ), to $1.12 \pm 0.56 \mathrm{~cm}^{2}$ at postoperative 24 months follow up (P $=0.048)$. These results were consistent with that reported in the study by Calafiore et al., [8], where in the treatment group, tenting area decreased significantly from $1.4 \pm 0.7 \mathrm{~cm}^{2}$ preoperatively to $0.7 \pm 0.3 \mathrm{~cm}^{2}$ postoperatively $(\mathrm{P}<0.001)$.

Fukuda and colleages [14] introduced an interesting concept, because they found preoperative tenting area (cutoff value, $0.80 \mathrm{~cm}^{2}$ ) to be risk factors for FTR. They emphasized that the cause of TR return could be a ventricular factor and not technical failure. They developed a new term called TV coaptation depth assessment, which can help with tricuspid surgery decision-making.

Our preoperative PAP was $45.9 \pm 6.27 \mathrm{mmHg}$ with significant decrease to 35.5 $\pm 4.5 \mathrm{mmHg}$ after one month and to $33 \pm 5 \mathrm{mmHg}$ after two years. Our results were higher than those reported in a study done by Chikwe et al., [15]. They reported that PAP for treated group with annuloplasty was $37.6 \pm 14.4 \mathrm{mmHg}$. This difference can be explained as we had higher incidence of atrial fibrillation resulting in a rise in PAP.

In a retrospective study involving 638 patients over a mean follow-up span of 64 months, Song and colleagues [16] found that 7.7\% of untreated mild TR progressed to late significant TR despite effective left-sided valve surgery. They proposed that in patients with late severe TR, aggressive surgical intervention could be considered because it was linked to worse clinical outcomes.

We agree with Navia and colleagues [17] and Kim et al., [18] who confirmed that moderate TR due to mitral valve diseases should be treated to improve patient's outcomes by giving benefit of doubt to prevent regurgitation progression and RV dysfunction.

\section{Limitations}

Limitations of this study may be: A retrospective design, small sample size, and difficult assessment of post-operative medications as diuretics on the severity of TR. 


\section{Conclusion}

We highly recommend repair for moderate tricuspid regurgitation during mitral valve surgery to avoid persistence or progression of the tricuspid regurgitation. We emphasize that correction of STR was able to reduce the grade of TR two years after surgery and improved right ventricular functions.

\section{Conflicts of Interest}

The authors declare no conflicts of interest regarding the publication of this paper.

\section{References}

[1] Nath, J., Foster, E. and Heidenreich, P.A. (2004) Impact of Tricuspid Regurgitation on Long-Term Survival. Journal of the American College of Cardiology, 43, 405-409. https://doi.org/10.1016/j.jacc.2003.09.036

[2] Dreyfus, G.D., Corbi, P.J., Chan, K.J. and Bahrami, T. (2005) Secondary Tricuspid Regurgitation or Dilatation: Which Should Be the Criteria for Surgical Repair? The Annals of Thoracic Surgery, 79, 127-132. https://doi.org/10.1016/j.athoracsur.2004.06.057

[3] Topilsky, Y., Nkomo, V.T., Vatury, O., Michelena, H.I., Letourneau, T., Suri, R.M., et al. (2014) Clinical Outcome of Isolated Tricuspid Regurgitation. JACC: Cardiovascular Imaging, 7, 1185-1194. https://doi.org/10.1016/j.jcmg.2014.07.018

[4] Mutlak, D., Aronson, D., Lessick, J., Reisner, S.A., Dabbah, S. and Agmon, Y. (2009) Functional Tricuspid Regurgitation in Patients with Pulmonary Hypertension: Is Pulmonary Artery Pressure the Only Determinant of Regurgitation Severity? Chest, 135, 115-121. https://doi.org/10.1378/chest.08-0277

[5] Filsoufi, F., Anyanwu, A.C., Salzberg, S.P., Frankel, T., Cohn, L.H. and Adams, D.H. (2005) Long-Term Outcomes of Tricuspid Valve Replacement in the Current Era. The Annals of Thoracic Surgery, 80, 845-850. https://doi.org/10.1016/j.athoracsur.2004.12.019

[6] Lang, R.M., Badano, L.P., Mor-Avi, V., Afilalo, J., Armstrong, A., Ernande, L., et al. (2015) Recommendations for Cardiac Chamber Quantification by Echocardiography in Adults: An Update from the American Society of Echocardiography and the European Association of Cardiovascular Imaging. European Heart Journal-Cardiovascular Imaging, 16, 233-271. https://doi.org/10.1093/ehjci/jev014

[7] Yilmaz, O., Suri, R.M., Dearani, J.A., Sundt III, T.M., Daly, R.C., Burkhart, H.M., et al. (2011) Functional Tricuspid Regurgitation at the Time of Mitral Valve Repair for Degenerative Leaflet Prolapse: The Case for a Selective Approach. The Journal of Thoracic and Cardiovascular Surgery, 142, 608-613.

https://doi.org/10.1016/j.jtcvs.2010.10.042

[8] Calafiore, A.M., Iacò, A.L., Romeo, A., Scandura, S., Meduri, R., Varone, E., et al. (2011) Echocardiographic-Based Treatment of Functional Tricuspid Regurgitation. The Journal of Thoracic and Cardiovascular Surgery, 142, 308-313. https://doi.org/10.1016/j.jtcvs.2010.04.047

[9] Hamdy, Y., Mostafa, M.M. and Elminshawy, A. (2019) DeVega versus Ring Annuloplasty in Severe Functional Tricuspid Insufficiency and Their Impact on the Right Ventricle. The Egyptian Cardiothoracic Surgeon, 1, 133-139.

https://doi.org/10.35810/ects.v1i4.89 
[10] Saran, N. and Dearani, J.A. (2018) Tricuspid Valve Repair: How I Teach It. The Annals of Thoracic Surgery, 105, 675-679. https://doi.org/10.1016/j.athoracsur.2017.08.063

[11] Ren, W.J., Zhang, B.G., Liu, J.S., Qian, Y.J. and Guo, Y.Q. (2015) Outcomes of Tricuspid Annuloplasty with and without Prosthetic Rings: A Retrospective Follow-Up Study. Journal of Cardiothoracic Surgery, 10, 1-7. https://doi.org/10.1186/s13019-015-0281-2

[12] Desai, R.R., Abello, L.M.V., Klein, A.L., Marwick, T.H., Krasuski, R.A., Ye, Y., et al. (2013) Tricuspid Regurgitation and Right Ventricular Function after Mitral Valve Surgery with or without Concomitant Tricuspid Valve Procedure. The Journal of Thoracic and Cardiovascular Surgery, 146, 1126-1132.

https://doi.org/10.1016/j.jtcvs.2012.08.061

[13] Agricola, E., Asmarats, L., Maisano, F., Cavalcante, J.L., Liu, S. and Milla, F. (2021) Imaging for Tricuspid Valve Repair and Replacement. Cardiovascular Imaging, 14, 61-111. https://doi.org/10.1016/j.jcmg.2020.01.031

[14] Fukuda, S., Gillinov, A.M., McCarthy, P.M., Stewart, W.J., Song, J.M., Kihara, T., et al. (2006) Determinants of Recurrent or Residual Functional Tricuspid Regurgitation after Tricuspid Annuloplasty. Circulation, 114, 1-582. https://doi.org/10.1161/CIRCULATIONAHA.105.001305

[15] Chikwe, J., Itagaki, S., Anyanwu, A. and Adams, D.H. (2015) Impact of Concomitant Tricuspid Annuloplasty on Tricuspid Regurgitation, Right Ventricular Function, and Pulmonary Artery Hypertension after Repair of Mitral Valve Prolapse. Journal of the American College of Cardiology, 65, 1931-1938. https://doi.org/10.1016/j.jacc.2015.01.059

[16] Song, H., Kim, M.J., Chung, C.H., Choo, S.J., Song, M.G., Song, J.M., et al. (2009) Factors Associated with Development of Late Significant Tricuspid Regurgitation after Successful Left-Sided Valve Surgery. Heart, 95, 931-936. https://doi.org/10.1136/hrt.2008.152793

[17] Navia, J.L., Brozzi, N.A., Klein, A.L., Ling, L.F., Kittayarak, C., Nowicki, E.R., et al. (2012) Moderate Tricuspid Regurgitation with Left-Sided Degenerative Heart Valve Disease: To Repair or Not to Repair? The Annals of Thoracic Surgery, 93, 59-69. https://doi.org/10.1016/j.athoracsur.2011.08.037

[18] Kim, J.B., Yoo, D.G., Kim, G.S., Song, H., Jung, S.H., Choo, S.J., et al. (2012) Mild-to-Moderate Functional Tricuspid Regurgitation in Patients Undergoing Valve Replacement for Rheumatic Mitral Disease: The Influence of Tricuspid Valve Repair on Clinical and Echocardiographic Outcomes. Heart, 98, 24-30. https://doi.org/10.1136/heartjnl-2011-300403 